\title{
Robustness of life histories to environmental variability in complex versus simple life cycles
}

\author{
Annie Jonsson ${ }^{1}$ (D) \\ Received: 25 March 2020 / Accepted: 8 January 2021 / Published online: 29 January 2021 \\ (c) The Author(s) 2021
}

\begin{abstract}
Most animal species have a complex life cycle (CLC) with metamorphosis. It is thus of interest to examine possible benefits of such life histories. The prevailing view is that CLC represents an adaptation for genetic decoupling of juvenile and adult traits, thereby allowing life stages to respond independently to different selective forces. Here I propose an additional potential advantage of CLCs that is, decreased variance in population growth rate due to habitat separation of life stages. Habitat separation of pre- and post-metamorphic stages means that the stages will experience different regimes of environmental variability. This is in contrast to species with simple life cycles (SLC) whose life stages often occupy one and the same habitat. The correlation in the fluctuations of the vital rates of life stages is therefore likely to be weaker in complex than in simple life cycles. By a theoretical framework using an analytical approach, I have (1) derived the relative advantage, in terms of long-run growth rate, of CLC over SLC phenotypes for a broad spectrum of life histories, and (2) explored which life histories that benefit most by a CLC, that is avoid correlation in vital rates between life stages. The direction and magnitude of gain depended on life history type and fluctuating vital rate. One implication of our study is that species with CLCs should, on average, be more robust to increased environmental variability caused by global warming than species with SLCs.
\end{abstract}

Keywords Life histories $\cdot$ Population growth rate $\cdot$ Long-run growth rate $\cdot$ Environmental variability $\cdot$ Species life cycle $\cdot$ Discrete time model

\section{Introduction}

The majority of animal species have complex life cycles (CLC) where individuals go through a metamorphosis involving a rapid and radical change in physiology, morphology, and ecology (Wilbur 1980; Werner 1988; Moran 1994; Truman and Riddiford 1999; Laudet 2011). Many species that today go through a radical and rapid morphological reconstruction during their life cycle, like amphibians and holometabolous insects, have ancestors whose development from juveniles to adults was of a more gradual nature (Szarski 1957; Brown 1977; Strathmann 1978; Truman and Riddiford 1999). Indeed, the major evolutionary trend in many taxa has been from simple life cycles (SLC) to CLC (Ebenman 1992; Truman and Riddiford 1999). Nevertheless, there are also species from various

Annie Jonsson

annie.jonsson@his.se

1 School of Bioscience, Ecological Modelling Group, University of Skövde, 54128 Skövde, Sweden taxa-including marine invertebrates and amphibians - that have eliminated metamorphosis, either through retention of larval traits in adults (paedomorphosis) or through direct development, and re-evolved a SLC (Wilbur and Collins 1973; Wassersug 1975; Strathmann 1978; Parks et al. 1988; Wray and Raff 1991; Bonett and Chippindale 2004; Wiens et al. 2007; Laudet 2011; Denoël and Ficetola 2014).

The search for explanations behind the evolutionary success of CLCs dates back nearly a century (Haldane 1932). The prevailing view is that CLCs represent a response to widespread selection for developmental independence of different stages of the life cycle of species. Such independence permits adaptive evolution within a life cycle stage without correlated negative effects on traits of other stages (Werner 1988; Ebenman 1992; Moran 1994; Truman and Riddiford 1999; Aguirre et al. 2014; Sherratt et al. 2017). The adaptive decoupling hypothesis for the evolution of complex life cycles with metamorphosis is based on the assumption that traits expressed in pre-metamorphic and post-metamorphic stages should be at least partially 
genetically uncorrelated, which has been observed to be the case (e.g., Phillips 1998; Watkins 2001; Aguirre et al. 2014).

Here I propose an additional potential advantage of CLCs over SLCs; CLCs are likely to be less affected by the destabilizing forces of a stochastically varying environment than SLCs. In contrast to organisms having SLCs, the life stages in those having CLCs often occupy different habitats. For example, in many species of frogs, the tadpoles occupy aquatic habitats while the adults are terrestrial, and in barnacles, the larval stage is pelagic while the adult stage is sedentary and occupies tidal zones. Juveniles and adults will therefore, to some degree, experience different regimes of environmental variation. This could be expected to lead to a reduced covariance in the fluctuations of the vital rates of the life stages. All else being equal, reduced covariance in the vital rates of different life stages will in turn lead to reduced variance in the growth rate of a population (Tuljapurkar 1982) with consequences for the long-run growth rate (fitness) and extinction risk of a population (Tuljapurkar 1982; Lande and Orzack 1988; Benton and Grant 1996; Saeter and Engen 2015). The effect of a changed covariance, between the vital rates of life stages, on the variance of population growth rate depends on the sensitivities of the population growth rate to changes in the vital rates (Tuljapurkar 1982; Benton and Grant 1996). These sensitivities might differ among species with different types of life histories, e.g., semelparous (in this study defined as organisms that mostly reproduce once or sometimes a few times during their life span) versus iteroparous species (Benton and Grant 1999), suggesting that some species will benefit more from adopting a CLC than others.

Although covariance in the fluctuations of vital rates is an important component of the variance in the growth rate of a population, the variances in the vital rates themselves are also of importance (Tuljapurkar 1982). If the environmental variability in the habitat occupied by one of the life stages of a species with a CLC increases and thereby causes increased variance in the vital rates of that life stage, this might select for the elimination of metamorphosis and the completion of the whole life cycle in the less variable habitat. The question is how much the variance has to increase in order to outweigh the positive effects of a reduced covariance on long-run growth rate.

I have here explored the role of variance and covariance in the fluctuations of vital rates of life stages for the long-run growth rate of CLC and SLC species. Using a discrete time mathematical model for the growth of a population with two life stages I investigated several scenarios, differing with respect to patterns of environmental variability and species' life histories (e.g., semelparous vs iteroparous species). For each variability scenario and life history, I analytically derived the relative advantage, in terms of long-run growth rate of CLCs compared with SLCs and vice versa.

\section{Methods}

\section{Model and analysis}

To compare the long-run growth rates in organisms with simple and complex life cycles living in a variable environment, I used a discrete time model of a population with two life stages (Jonsson and Ebenman 2001):

$N_{1}(t+1)=s_{1}(t)(1-g(t)) N_{1}(t)+f(t) N_{2}(t)$

$N_{2}(t+1)=s_{1}(t) g(t) N_{1}(t)+s_{2}(t) N_{2}(t)$

Here $N_{1}$ is the number of juveniles and $N_{2}$ is the number of adults; $s_{1}$ and $s_{2}$ are the survival probabilities of juveniles and adults, respectively; $g$ is the probability of maturation; and $f$ is the fecundity of adults. The model describes the case where individuals must reach a certain stage to be able to reproduce. The model can also be presented in matrix form:

$\mathbf{N}(t+1)=\mathbf{A}(t) \mathbf{N}(t)$

where

$\mathbf{A}(t)=\left[\begin{array}{cc}s_{1}(t)(1-g(t)) & f(t) \\ s_{1}(t) g(t) & s_{2}(t)\end{array}\right]$

and

$\mathbf{N}(t)=\left[\begin{array}{l}N_{1}(t) \\ N_{2}(t)\end{array}\right]$

The dominant eigenvalue of the transition matrix $A$ at time $t$ is the population growth rate at time $t$. The average population growth rate, here, can be calculated from a matrix with mean values of vital rates. In serially independent environments, the stochastic long-run growth rate of the population, $\ln \lambda_{s}$, can be approximated by (Tuljapurkar 1982)

$\ln \lambda_{s} \approx \ln \lambda_{1}-\frac{\operatorname{Var}\left(\lambda_{1}\right)}{2 \lambda_{1}^{2}}$

Further, the linear approximation for $\operatorname{Var}\left[\lambda_{1}\right]$ in the case of two varying vital rates (with mean values $\overline{z_{1}}$ and $\overline{z_{2}}$ ) is (Tuljapurkar 1982):

$\operatorname{Var}\left[\lambda_{1}\left(\overline{z_{1}}, \overline{z_{2}}\right)\right] \approx \sum_{i=1}^{2} \operatorname{Var}\left(\overline{z_{i}}\right)\left(\frac{\partial \lambda_{1}}{\partial \overline{z_{1}}}\right)^{2}+2\left[\operatorname{Cov}\left(\overline{z_{1}}, \overline{z_{2}}\right)\right] \frac{\partial \lambda_{1}}{\partial \overline{z_{1}}} \frac{\partial \lambda_{1}}{\partial \overline{a_{2}}}$

Here $\frac{\partial \lambda_{1}}{\partial \bar{z}_{i}}$ is the sensitivity of $\lambda_{1}$ to a small absolute change in the vital rate $\overline{z_{\mathrm{i}}}$. Now, since the mean values of vital rates may differ by order of magnitudes, I replace sensitivities 
with elasticities $e\left(\overline{z_{i}}\right)=\frac{\partial \lambda_{1}}{\partial \bar{z}_{i}} \frac{\overline{z_{i}}}{\lambda_{1}}$ (the effect of a proportional change in the vital rate $\overline{z_{i}}$ on $\lambda_{1}$ ). Equation (6) can then be rewritten as
The mean fecundity for a given life history can be derived from the characteristic equation of the mean matrix and is then given by

$\operatorname{Var}\left[\lambda_{1}\left(\overline{z_{1}}, \overline{z_{2}}\right)\right] \approx \sum_{i=1}^{2}\left\{\lambda_{1} e\left(\overline{z_{\mathrm{i}}}\right) C V\left(\overline{z_{\mathrm{i}}}\right)\right\}^{2}+c\left(\overline{z_{1}}, \overline{z_{2}}\right) 2 \lambda_{1}^{2} e\left(\overline{z_{1}}\right) C V\left(\overline{z_{1}}\right) e\left(\overline{z_{2}}\right) C V\left(\overline{z_{2}}\right)$

Here $c\left(\overline{z_{1}}, \overline{z_{2}}\right)$ is the correlation in the fluctuations of the vital rates, and $C V\left(\overline{z_{\mathrm{i}}}\right)$ is the coefficient of variation for vital rate $\overline{z_{\mathrm{i}}}$. From Eq. (7) one can conclude that a positive correlation always increases, and a negative one always decreases the variance in population growth rate relative to zero correlation. Note that the effect of correlation on variance in growth rate depends on the elasticity of $\lambda_{1}$ to changes in the vital rates. These elasticities differ among organisms with different life histories. I have therefore explored a broad range of life histories to include differences.

\section{Life histories and parameter settings}

Two important characteristics of life histories are the developmental (maturation) rate and the reproductive strategy. Development to maturity can be rapid (precocious) or delayed; organisms may reproduce only once (semelparous) or iterated times (iteroparous). Further, for a given reproductive effort, an organism may produce several small offspring or a few large ones. A fundamental premise in most studies of life history evolution is that evolution is constrained by trade-offs between different vital rates (e.g. Roff 1992; Charlesworth 1994). In order to account for this, I have set the average growth rate $\left(\lambda_{1}\right)$ to be equal for all the different life histories and assumed three trade-offs that all include fecundity (Roff 1992):

(i) a cost of reproduction leading to a trade-off between adult survival and fecundity (that is, a high $s_{2}$ means a low $f$ and vice versa),

(ii) a trade-off between number and size of offspring, combined with increased juvenile survival with offspring size, leading to a trade-off between juvenile survival and fecundity (that is, a high $s_{1}$ means a low $f$ and a low $s_{1}$ means a high $f$ and vice versa)

(iii) increased body size of adults with decreasing developmental rate, combined with increased fecundity with body size, leading to a trade-off between developmental rate and fecundity (that is, a low $g$ means a high $f$ and vice versa). $f=\frac{\lambda_{1}^{2}+\left(s_{1} g-s_{1}-s_{2}\right) \lambda_{1}+s_{1} s_{2}(1-g)}{s_{1} g}$

Mean values of the vital rates and their elasticities for the eight examined life histories are given in Table 1. Assuming that there is an extra cost for a CLC, due to metamorphosis (Geffen et al. 2007), that overweighs other gains, such as escape intraspecific competition between stages or predators or attain a more effective resource use (McMahon and Hayward 2016) I also examined calculations that included a $1 \%$ reduce in fecundity, deriving from the reasoning that the extra cost might have an impact on adult size or on reproductive organs that further lead to lower fecundity (that is same size of offspring but fewer) or lower juvenile survival (the same amount of offspring but smaller sizes). Reduced fecundity affect elasticity values as well as the dominant eigenvalue $\lambda_{1}$.

Table 1 Mean values of the vital rates and their elasticities for the eight different life histories examined. Due to the trade-off between fecundity, $f$, and the other vital rates $\left(s_{1}, s_{2}\right.$, and $\left.g\right)$ in combination with the constraint of equal lambda $\left(\lambda_{1}=2.78\right)$ for all life histories, the value of fecundity varies and is given by Eq. $8 . \mathrm{S}=$ semelparous (however not in the strict sense, i.e., $s_{2}=0$, since there is a small probability of adult survival, $\left.s_{2}=0.1\right), \mathrm{I}=$ iteroparous, $\mathrm{D}=$ delayed maturation, $\mathrm{P}=$ precocious maturation, $\mathrm{M}=$ many small offspring, and $\mathrm{F}=$ few large offspring

\begin{tabular}{llllll}
\hline Life history & $z_{j}$ & $s_{1}$ & $s_{2}$ & $g$ & $f$ \\
\hline SDM & $\overline{z_{j}}$ & 0.001 & 0.1 & 0.1 & 74,480 \\
& $e\left(\overline{z_{j}}\right)$ & 0.4909 & 0.0183 & 0.4907 & 0.4908 \\
SDF & $\overline{z_{j}}$ & 0.9 & 0.1 & 0.1 & 58.7 \\
& $e\left(\overline{z_{j}}\right)$ & 0.5763 & 0.0152 & 0.3898 & 0.4084 \\
SPM & $\overline{z_{j}}$ & 0.001 & 0.1 & 0.9 & 8.278 \\
& $e\left(\overline{z_{j}}\right)$ & 0.4909 & 0.0183 & 0.4907 & 0.4908 \\
SPF & $\overline{z_{j}}$ & 0.9 & 0.1 & 0.9 & 8.9 \\
& $e\left(\overline{z_{j}}\right)$ & 0.4991 & 0.0180 & 0.3375 & 0.4829 \\
IDM & $\overline{z_{j}}$ & 0.001 & 0.9 & 0.1 & 52.247 \\
& $e\left(\overline{z_{j}}\right)$ & 0.4035 & 0.1931 & 0.4034 & 0.4034 \\
IDF & $\overline{z_{j}}$ & 0.9 & 0.9 & 0.1 & 41.2 \\
& $e\left(\overline{z_{j}}\right)$ & 0.4883 & 0.1657 & 0.3302 & 0.3460 \\
IPM & $\overline{z_{j}}$ & 0.001 & 0.9 & 0.9 & 5.807 \\
& $e\left(\overline{z_{j}}\right)$ & 0.4034 & 0.1931 & 0.4033 & 0.4034 \\
IPF & $\overline{z_{j}}$ & 0.9 & 0.9 & 0.9 & 6.2 \\
& $e\left(\overline{z_{j}}\right)$ & 0.4114 & 0.1906 & 0.2782 & 0.3981 \\
\hline
\end{tabular}


I calculated the long-run growth rate $\left(\ln \lambda_{s}\right)$ and the variance in growth rate $\left(\operatorname{Var}\left[\lambda_{1}\right]\right)$ for the eight different life histories in Table 1, and for different degrees of environmental variability in the habitats occupied by these life stages. I investigated three patterns of environmental variability for CLCs: equal variability in the juvenile and adult habitats, higher variability in the juvenile habitat than in the adult habitat, and vice versa. The life stages of SLCs were exposed to one and the same habitat with low respectively high variability. Organisms with CLCs (with juveniles and adults in different habitats) were assumed to have zero environmental correlation $(c=0)$ and SLCs (with juveniles and adults in the same habitat) with positive correlation $(c=1)$. Environmental variability was assumed to affect one vital rate in each stage that is, either fluctuating juvenile and adult survival $\left(s_{1}, s_{2}\right)$, juvenile survival and fecundity $\left(s_{1}, f\right)$, maturation rate and adult survival $\left(g, s_{2}\right)$, or maturation rate and fecundity $(g, f)$. Figure 1 schematically outlines the analysed scenarios. For all scenarios, except CLCs with a cost, the dominant eigenvalue of the average matrix $\left(\lambda_{1}\right)$ was set to 2.78 guaranteeing a longrun growth rate higher than unity for all life histories with zero correlation between vital rates. For scenarios where CLCs had a cost, the dominant eigenvalue was lower than 2.78.

\section{Results}

The most robust class of life histories, in terms of highest long-run growth rates are, as expected, complex life histories (CLCs), without a cost of metamorphosis, living in low variable habitats (Fig. 2). This result is due to the assumptions made in the model, a CLC will thus always have a higher long term growth rate compared with a corresponding SLC; however, the magnitude of the benefit depends on the lifehistory and the amount of variation compared with SLC. Life histories living in a high variable habitat will benefit most by a CLC strategy. However, the magnitude of gain depends on life history type and fluctuating vital rate. Furthermore, there is a larger benefit of a CLC strategy in comparison with a SLC if the juvenile stage experiences lower variable environment than the adult stage.

Figure 3 and 4 show specified comparisons from the calculations of long-run growth rates for the different scenarios and strategies a-d in Fig. 1. Note that in the case of the scenarios in Fig. 4, a SLC life history will experience a twofold cost, that is higher variability for one of the stages and correlated vital rates, compared with the CLC. That is due to the CLC benefit of being able to place one stage in

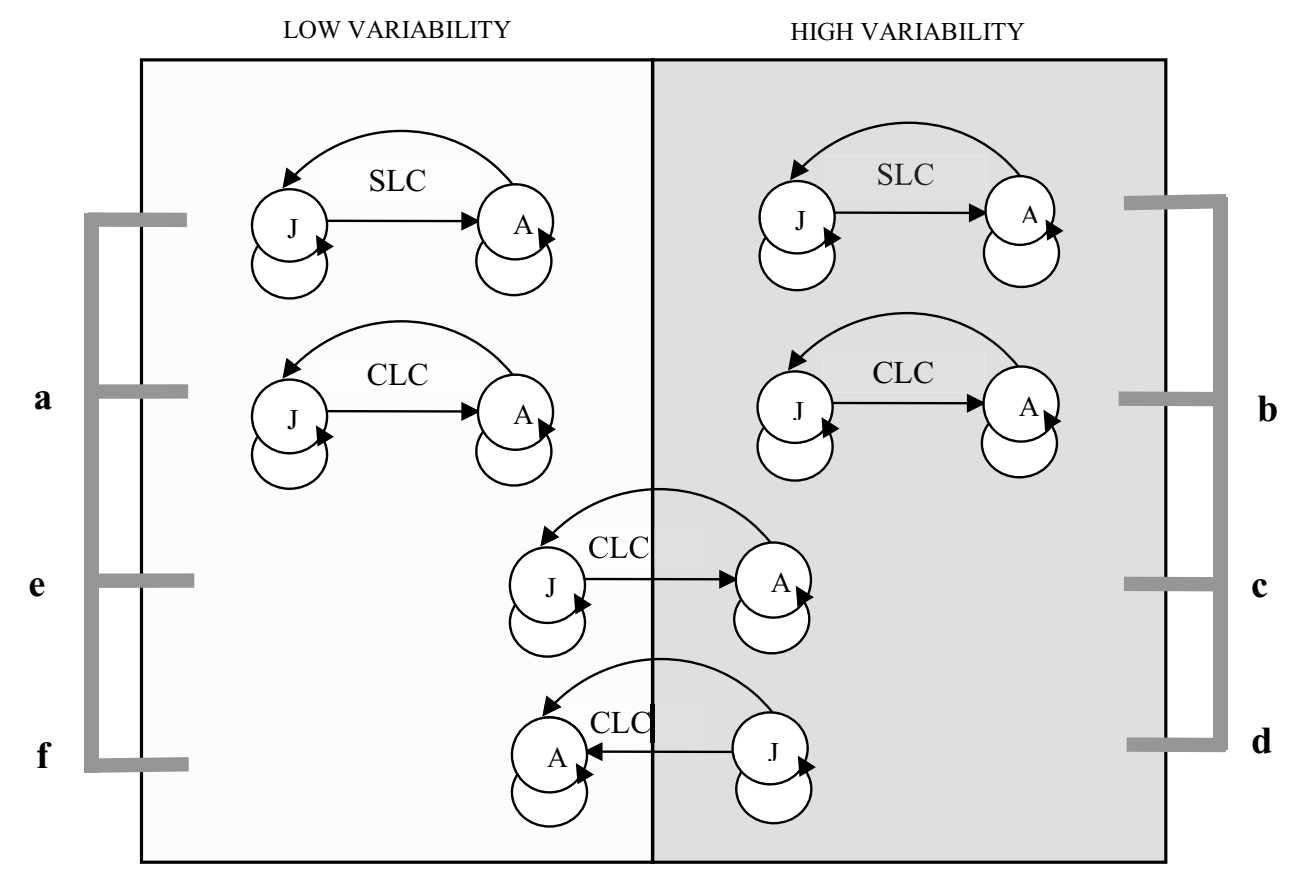

Fig. 1 Conceptual picture showing the investigated scenarios of simple (SLC) and complex life cycle (CLC) species, occupying one or two habitats, respectively. Light and dark grey colours illustrate the difference in magnitude of environmental variation where light grey represents low variability and dark grey larger variability. SLC species are assumed to have a positive correlation $(c=1)$ in the variation between vital rates in juveniles and adults, while CLC species are assumed to have zero corre- lation $(c=0)$. In circles $\mathrm{J}=$ juvenile stage and $\mathrm{A}=$ adult stage. Results for calculations of strategies $\mathbf{a}-\mathbf{b}$ are shown in Fig. 3 and for strategies $\mathbf{c}-\mathbf{d}$ in Fig. 4. Strategies $\mathbf{e}-\mathbf{f}$ represents potential beneficial strategies depending on variability in the grey habitat and results on examples of critical values of variability for the strategies to be beneficial are discussed and presented in Table 2 


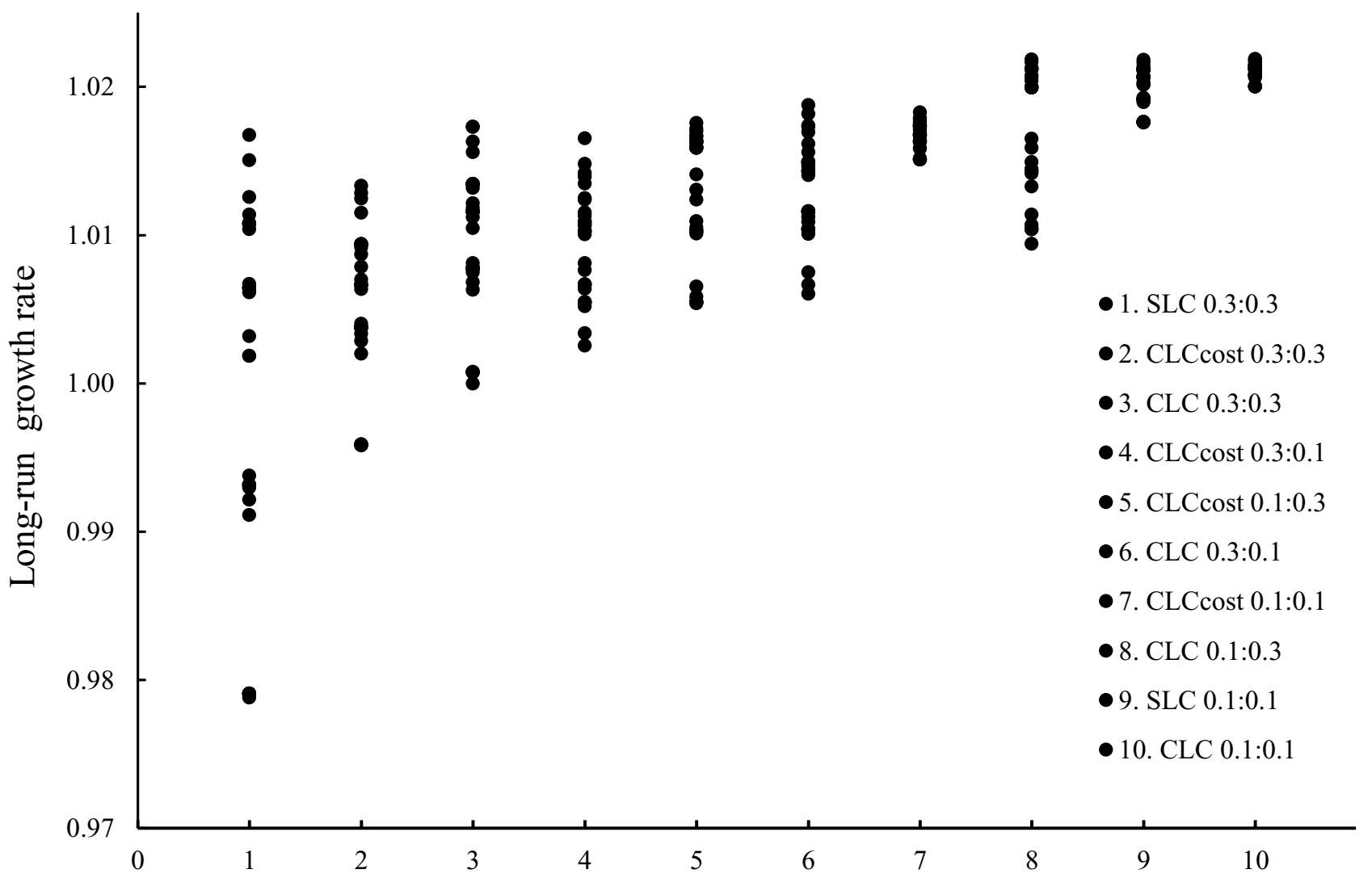

Fig. 2 Long-run growth rates for the different life histories for all the examined environmental scenarios described in the method. SLC $=$ simple life cycle, CLC $=$ complex life cycle, and CLCcost $=$ complex life cycle with a cost of metamorphosis. First value in

a less variable environment. A species with a CLC without a cost always had a higher long-run growth rate than a corresponding species with a SLC. The most advantageous life histories, when comparing a CLC strategy with a SLC strategy, were semelparous life histories with fluctuating juvenile survival and fecundity, strategies $\mathrm{c}$ and d (Fig. 4b, f), and semelparous life histories with many offspring and fluctuating maturation rate and fecundity, strategies $\mathrm{c}$ and $\mathrm{d}$ (Fig. 4d, h). On the other hand, semelparous life histories with fluctuating maturation rate and adult survival as well as semelparous life histories with fluctuating juvenile and adult survival in strategies $b$ and $d$ benefit the least (Figs. 3 and 4e). For CLC species including a cost of metamorphosis (Figs. 3 and 4, black bars) the calculated examples showed that no life history benefited by a CLC strategy in the low variable environment (Fig. 3a-d; strategy a in Fig. 1). Neither did semelparous life histories with fluctuating adult survival, with juveniles in high variable habitat, benefit (Figs. 3 and 4e, g; strategies $b$ and $c$ in Fig. 1).

A species with a CLC may have an advantage even if one of the life stages occupies a more variable habitat than the corresponding life stage of a SLC (Fig. 1 strategies e-f). legend, 0.1 or 0.3 , is $C V$ of juvenile stage and second value, 0.1 or 0.3 , is $C V$ of adult stage. Each of the ten cases show 32 values (dots) that represent the calculations for 8 life histories times 4 combinations of fluctuating vital rates

Setting the long-run growth rates of a SLC and a CLC to be equal one can solve for the critical variability, $C V\left(\overline{z_{\mathrm{j}}}\right)$, below which the CLC will have an advantage and above which the SLC will be favoured:

$\mathrm{CV}\left(z_{j}\right)=\frac{C V\left(\overline{z_{i}}\right)\left[e\left(\overline{z_{j}}\right) \cdot\left(e\left(\overline{z_{j}}\right)+2 c\left(\overline{z_{i}}, \overline{z_{j}}\right) e\left(\overline{z_{i}}\right)\right)\right]^{1 / 2}}{e\left(\overline{z_{j}}\right)}$

It can be seen that positive correlation between the vital rates of the life stages in SLC species (juveniles and adults in the same habitat) results in higher permissible $C V\left(\overline{z_{\mathrm{j}}}\right)$ for a life stage of a corresponding CLC (occupying a separate habitat). Moreover, the lower the elasticity, $e\left(\overline{z_{\mathrm{j}}}\right)$, for that stage, the higher the allowable $C V\left(\overline{z_{\mathrm{j}}}\right)$. In Table 2 I show examples of calculations of critical values for the scenarios where a SLC, in a habitat with a variation of $C V=0.1$, could benefit, that is having a higher long-run growth rate, if it had a complex life cycle (without cost of metamorphosis), even if one of the life stages was in a more variable environment.

Semelparous organisms $\left(s_{2}=0.1\right)$ distinguish by having a very low elasticity with respect to adult survival (Table 1 ). 


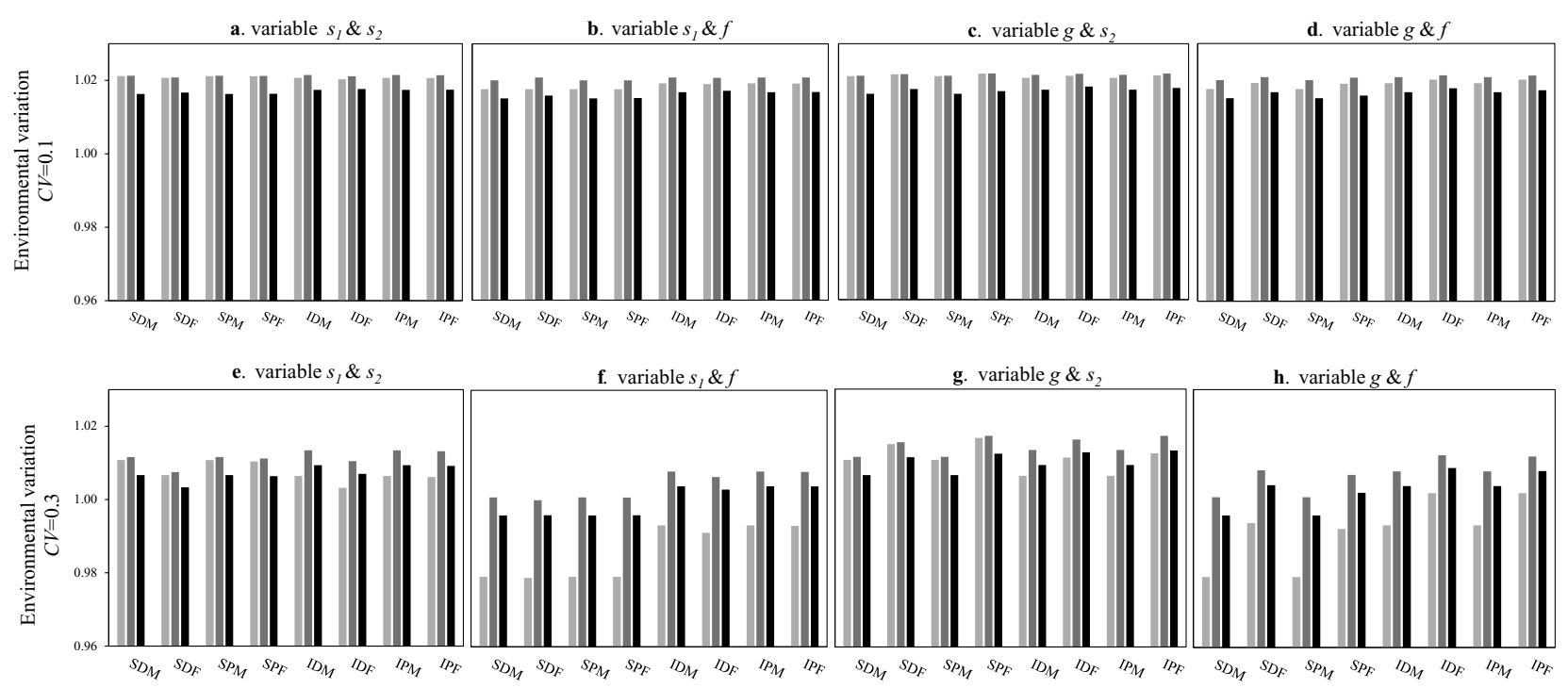

Fig. 3 Long-run growth rates ( $\ln \lambda_{s}$ ) for eight SLCs (light grey bars) and eight CLCs without cost (dark grey bars) and with a cost of $1 \%$ in fecundity (black bars). Magnitude of environmental variability is the same in both stages that is, $C V=0.1$ in A-D (upper row), which represent strategy a in Fig. 1 , and $C V=0.3$ in $\mathrm{E}-\mathrm{H}$ (bottom row), which represents strategy $b$ in Fig. 1. Environmental variability

This means an advantage for a semelparous CLC species to have the adult stage placed in a separate habitat even if this habitat implies a substantial increase in $C V\left(s_{2}\right)$ as seen in Table 2. On the other hand, semelparous organisms affects in $\mathbf{a}$ and $\mathbf{e} s_{1}, s_{2} ; \mathbf{b}$ and $\mathbf{f} s_{1}, f ; \mathbf{c}$ and $\mathbf{g} g, s_{2}$; and $\mathbf{d}$ and $\mathbf{h} g$, $f$, where $s_{1}=$ juvenile survival, $s_{2}=$ adult survival, $g=$ maturation rate, and $f=$ fecundity. Abbreviation for life history type: $\mathrm{I}=$ iteroparous $\left(s_{2}=0.9\right)$ or $\mathrm{S}=$ semelparous $\left(s_{2}=0.1\right), \mathrm{P}=$ precocious $(g=0.9)$ or $\mathrm{D}=$ delayed $(g=0.1), \mathrm{F}=$ few large offspring or $\mathrm{M}=$ many small offspring. Correlation in variability is $c=1$ for SLC and $c=0$ for CLC

have high elasticities with respect to juvenile survival which means that there is no advantage of a semelparous CLC having juveniles in a separate habitat if that habitat implies a high variation in juvenile survival, $C V\left(s_{1}\right)$.

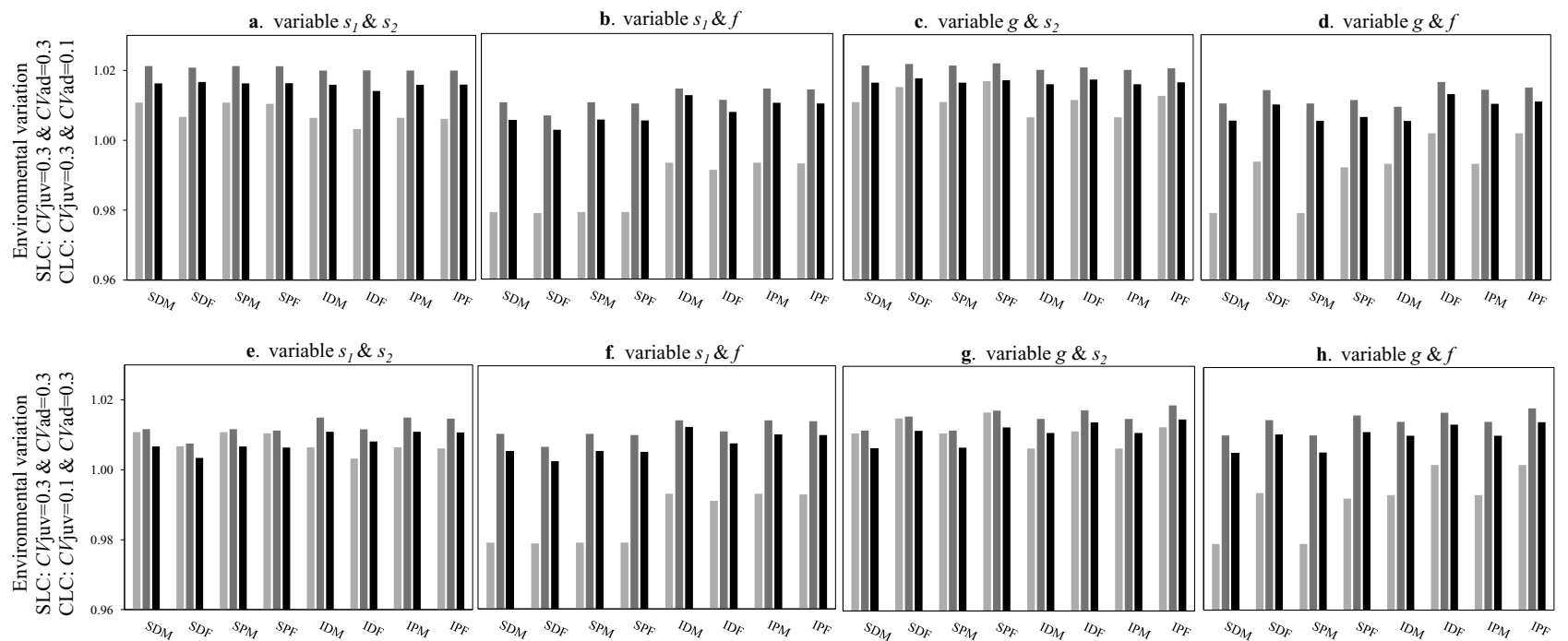

Fig. 4 Long-run growth rates $\left(\ln \lambda_{s}\right.$ ) for eight simple life cycle (light grey bars) and eight complex life cycle without cost (dark grey bars) and with a cost of $1 \%$ in fecundity (black bars). Magnitude of environmental variability is the same for both stages in SLCs, that is, $C V=0.3$, but differ for CLCs that is $C V=0.1$ for juveniles and $C V=0.3$ for adults in a-d (that is low juvenile and high adult variability in upper row), representing strategy $\mathrm{c}$ or e in Fig. 1, and $C V=0.3$ for juveniles and $C V=0.1$ for adults in $\mathbf{e}-\mathbf{h}$ (that is high juvenile and low adult variability in bottom row), representing strategy $\mathrm{d}$ in Fig. 1. Environmental variability affects in $\mathbf{a}$ and $\mathbf{e} s_{1}, s_{2}$; b and $\mathbf{f} s_{1}, f$; $\mathbf{c}$ and $\mathbf{g} g, s_{2}$; and $\mathbf{d}$ and $\mathbf{h} g, f$; where $s_{1}=$ juvenile survival, $s_{2}=$ adult survival, $g=$ maturation rate, and $\mathrm{f}=$ fecundity. Abbreviation for life history type: $\mathrm{I}=$ iteroparous $\left(s_{2}=0.9\right)$ or $\mathrm{S}=$ semelparous $\left(s_{2}=0.1\right), \mathrm{P}=$ precocious $(g=0.9)$ or $\mathrm{D}=$ delayed $(g=0.1), \mathrm{F}=$ few large offspring or $\mathrm{M}=$ many small offspring. Correlation in variability is $c=1$ for SLC and $c=0$ for CLC 
Table 2 Critical values for the coefficient of variation below which a complex life cycle without cost (CLC) still is beneficial in comparison with a simple life cycle (SLC) even if the stage in low variable environment $(C V=0.1)$ were in a higher variable environment (see strategies e-f in Fig. 1). Variability is either on $s_{1}$ and $s_{2}$ or $s_{1}$ and $f$. Above the critical value a SLC has a higher long-run growth rate than a CLC. Abbreviation for life history type: $\mathrm{I}=$ iteroparous $\left(s_{2}=0.9\right)$, $\mathrm{S}=$ semelparous $\quad\left(s_{2}=0.1\right), \quad \mathrm{P}=$ precocious $\quad(g=0.9), \quad \mathrm{D}=$ delayed $(g=0.1), \mathrm{F}=$ few large offspring and $\mathrm{M}=$ many small offspring. See text and Table 1 for further explanations and specifications. Correlation in variability is $c=1$ for SLC and $c=0$ for CLC

\begin{tabular}{llllll}
\hline Life history & \multicolumn{2}{l}{ Variable $s_{1}$ and $s_{2}$} & & \multicolumn{2}{l}{ Variable $s_{1}$ and $f$} \\
\cline { 2 - 3 } \cline { 6 - 6 } & $\begin{array}{l}\mathrm{CV}\left(s_{1}\right) \text { for } \\
\text { transition } \mathrm{F}\end{array}$ & $\begin{array}{l}\mathrm{CV}\left(s_{2}\right) \text { for } \\
\text { transition } \mathrm{E}\end{array}$ & & $\begin{array}{l}\mathrm{CV}\left(s_{1}\right) \text { for } \\
\text { transition } \mathrm{F}\end{array}$ & $\begin{array}{l}\mathrm{CV}(f) \text { for } \\
\text { transition } \mathrm{E}\end{array}$ \\
\hline $\mathrm{SDM}$ & 0.1037 & 0.7390 & & 0.1732 & 0.1732 \\
$\mathrm{SDF}$ & 0.1026 & 0.8754 & & 0.1554 & 0.1955 \\
$\mathrm{SPM}$ & 0.1037 & 0.7389 & & 0.1732 & 0.1732 \\
$\mathrm{SPF}$ & 0.1035 & 0.7510 & & 0.1751 & 0.1713 \\
$\mathrm{IDM}$ & 0.1399 & 0.2275 & & 0.1732 & 0.1732 \\
$\mathrm{IDF}$ & 0.1296 & 0.2626 & & 0.1554 & 0.1955 \\
$\mathrm{IPM}$ & 0.1400 & 0.2276 & & 0.1732 & 0.1732 \\
$\mathrm{IPF}$ & 0.1388 & 0.2306 & 0.1751 & 0.1751 \\
\hline
\end{tabular}

From Eq. (7) it can be understood that the relative contribution of the correlation between vital rates of juveniles and adults to the variance of population growth rate has a maximum when:

$\frac{e\left(\overline{z_{j}}\right)}{e\left(\overline{z_{i}}\right)}=\frac{\operatorname{CV}\left(\overline{z_{i}}\right)}{\operatorname{CV}\left(\overline{z_{j}}\right)}$

Here $\mathrm{CV}\left(\overline{z_{i}}\right)$ and $\mathrm{CV}\left(\overline{z_{j}}\right)$ are the coefficients of variation for the vital rates $\overline{z_{i}}$ and $\overline{z_{j}}$ of the SLC life stages $i$ and $j$, respectively. If $j$ is the adult stage and $i$ is the juvenile stage $\overline{z_{j}}=s_{2}$ or $\overline{z_{j}}=f$ and $\overline{z_{i}}=s_{1}$ or $z_{i}=\mathrm{g}$, respectively. In the special case when the relative variability in the vital rates of juveniles and adults are equal (that is, $C V\left(\overline{z_{i}}\right)=C V\left(\overline{z_{j}}\right)$ ), the organism that are most sensitive to correlation are those with life histories where elasticities are equal (i.e., where $e\left(\overline{z_{i}}\right)=e\left(\overline{z_{j}}\right)$ ). For varying juvenile and adult survival, elasticities are more similar in organisms with iteroparous life histories than in those with semelparous life histories (Table 1). This means that SLC species with iteroparous life histories are particularly affected by correlation and as result have particularly low long-run growth rates compared with iteroparous species with CLC (Fig. 3a, e).

There are also scenarios where species with SLCs have higher long-run growth rate than those with CLCs. If the juvenile stage of a species with a CLC inhabits a much more variable environment than the juvenile stage of a species with a SLC (Fig. 1, strategy f), the species with SLC will have a higher long-run growth rate irrespective of its life history. Also, if the adult stage of a species with a CLC inhabits a much more variable environment than the adult stage of a species with SLC, the species with a SLC will have a higher long-run growth rate for many life history types (Fig. 1, strategy e).

\section{Discussion}

The genetic decoupling hypothesis for the evolution of complex life cycles (Werner 1988; Ebenman 1992; Moran 1994; Aguirre et al. 2014) and the "environmental decoupling" hypothesis proposed in the present work have one thing in common: the advantage of complex life cycles is that they lead to increased independence of the different life stages in the life cycle. The two hypotheses are not mutually exclusive, instead both are likely to apply in many cases. Indeed, the genetic decoupling of life stages might be necessary for environmental decoupling to be manifested. From this point of view, reduced covariance in the fluctuations of vital rates is an additional benefit following from genetic and adaptive decoupling - a bonus. This is also in line with the result of ten Brink et al (2019) where it is shown that environmental decoupling favours CLCs. Metamorphosis reduce tension between individuals competing for two food sources, where the secondary food source only is available to large individuals, by letting the individuals change their diet during their life cycle. An example that shows that complex life histories are a most successful strategy among animal species is that more than $60 \%$ of all described animal species are holometabolous (complete metamorphosis) insects, a strategy that evolved over 300 million years ago (McMahon and Hayward 2016). Using size-structured consumer-resource models, ten Brink et al.'s (2019, 2020) results support the success story of metamorphosis. ten Brink et al (2020) conclude that even if metamorphosis comes with costs and risks, the strategy of metamorphosis is not easily lost.

Focusing on the role of environmental decoupling of life stages, this study shows that the relative advantage, in terms of long-run growth rate, of complex life cycles over simple life cycles differs among species with different types of life histories and also depends on which vital rates that are most affected by environmental variability. This is because the life history of a species determines the elasticity of population growth rate to changes in the vital rates of life stages. For iteroparous species, for instance, a change in adult survival has a relatively large effect on population growth rate (i.e., high elasticity) compared with species with more semelparous life histories (low elasticity). Therefore, if environmental variation primarily affects survival rates of the life stages, this means that the advantage, in terms of long-run growth rate and persistence, of species with CLC over those with SLC 
will be larger for species with iteroparous life histories than for those with more semelparous life histories (all else being equal). On the other hand, changes in fecundity have a larger effect on population growth rate for species with semelparous life histories (i.e., high elasticities) than for those with iteroparous life histories. As a consequence, CLC species with semelparous life histories will have a larger advantage than those with iteroparous ones if environmental variation primarily affects fecundity.

These results also have a bearing on an interesting pattern found in many natural populations: an inverse relationship between the variance in a vital rate and the sensitivity of population growth rate to changes in that vital rate (Pfister 1998; Saether and Bakke 2000). Such a relationship decreases the variance in growth rate and hence increases long-run growth rate and decreases extinction risk of a population (Lande and Orzack 1988). One possible mechanism producing such a relationship would be if the life stages of a species occupy different habitats-which they often do in CLC species-and the life stage whose vital rates have the largest effect on population growth rate (highest elasticity) occupy a less variable habitat.

Although there are many advantages connected to a complex life cycle, several species show an evolutionary transition from CLC to SLC (Wilbur and Collins 1973; Wassersug 1975; Strathmann 1978; Parks et al. 1988; Wray and Raff 1991; Wiens et al. 2007; Laudet 2011; Denoël and Ficetola 2014). For species living in a variable environment, the long-run growth rate is the appropriate fitness measure over long periods of time (Saeter and Engen 2015). Thus, for organisms with CLC, with juveniles and adults living in different habitats, an increase in the environmental variability in one of the habitats might select for elimination of metamorphosis. That is, selection might favour completion of the entire life cycle in one and the same habitat, even if this leads to an increased covariance between the vital rates of juveniles and adults. Critical values of variability where it will become advantageous to evolve a SLC and complete the whole life cycle in one and the same habitat can be derived from Eq. (10) and are shown in Tab 2. An increase in variability of fecundity or adult survival above these values will select for paedomorphosis - the elimination of the post-metamorphic stage and retention of juvenile morphology in reproductive adults. Because iteroparous organisms have a higher elasticity with respect to adult survival than semelparous organisms, evolution of paedomorphosis could be expected to be more common among iteroparous organisms than among semelparous ones. An increase in variability of juvenile survival above the critical value will instead select for elimination of the pre-metamorphic stage (e.g., larval phase) and evolution of direct development. This could be expected to be more common among semelparous organisms than among iteroparous ones, since semelparous organisms have higher elasticity with respect to juvenile survival than iteroparous ones.
The elimination of metamorphosis and completion of the whole life cycle in one habitat is the most extreme change of a complex life cycle. Less drastic evolutionary modifications of complex life cycles, without a loss of metamorphosis, may involve the amount of time spent in the different life stages (Wilbur and Collins 1973; Werner 1986; Rowe and Ludwig 1991; Hentschel 1999). For instance, evolutionary changes in maturation rate will change the time spent in the juvenile habitat.

To conclude, this study provides a theoretical framework for comparing long-run growth rates of species with simple and complex life cycles. Using the framework, one find that species with complex life cycles will often have a higher long-run growth rate than species with simple life cycles. The reason for this is that the life stages of species with complex life cycles live in different habitats and as a result they are less likely to suffer from positive covariation in the vital rates of life stages. That is, complex life cycles with metamorphosis means increased independence of the different stages in the life cycle of a species. An important implication of the study is that species with CLCs should be less vulnerable to increased environmental variability, for example, caused by global warming, than species with SLCs.

Future work should incorporate and explore the effects of serial correlation (Tuljapurkar and Orzack 1980; Tuljapurkar 1982, 1990; Ruokolainen et al. 2009; Saether and Engen 2015) and density dependence in vital rates (Metz et al. 1992; Ferriere and Gatto 1995; Ebenman et al. 1996; Grant 1997; Saeter and Engen 2015). Density dependence can be caused by competition within and between life stages for limiting resources. Since genetic decoupling in species with CLC allows life stages to adapt to different niches, it will lead to reduced competition between life stages (Ebenman 1987, 1992). Decreased competition between life stages is likely to lead to increased population abundance due to reduced intraspecific competition (Schoener 1973) resulting in decreased extinction risk (Lande 1993). This suggests that CLC species might have an advantage over SLC species in a variable environment also in the presence of density dependence in the vital rates.

Acknowledgements The author thanks Bo Ebenman for discussions and support.

Funding Open Access funding provided by University of Skövde The project was funded by Linköping and Skövde University.

Availability of data and material The analytic expressions that are the bases for the study are clearly shown in the paper, and all calculated values are based on these expressions.

Code availability There is no specific code since calculations are simple mathematical calculations based on the presented analytical expressions. 
Open Access This article is licensed under a Creative Commons Attribution 4.0 International License, which permits use, sharing, adaptation, distribution and reproduction in any medium or format, as long as you give appropriate credit to the original author(s) and the source, provide a link to the Creative Commons licence, and indicate if changes were made. The images or other third party material in this article are included in the article's Creative Commons licence, unless indicated otherwise in a credit line to the material. If material is not included in the article's Creative Commons licence and your intended use is not permitted by statutory regulation or exceeds the permitted use, you will need to obtain permission directly from the copyright holder. To view a copy of this licence, visit http://creativecommons. org/licenses/by/4.0/.

\section{References}

Aguirre JD, Blows MW, Marshall DJ (2014) The genetic covariance between life cycle stages separated by metamorphosis. Proc R Soc B 281(1788):20141091

Benton TG, Grant A (1996) How to keep fit in the real world: elasticity analyses and selection pressures on life histories in a variable environment. Am Nat 147:115-139

Benton T, Grant A (1999) Elasticity analysis as an important tool in evolutionary and population ecology. Trends Ecol Evol 14:467-471

Bonett RM, Chippindale PT (2004) Speciation, phylogeography and evolution of life history and morphology in plethodontid salamanders of the Eurycea multiplicata complex. Mol Ecol 13:1189-1203

Brown VK (1977) Metamorphosis: a morphometric description. Int J Insect Morphol Embryol 6:221-223

Charlesworth B (1994) Evolution in age-structured populations. Cambridge University Press, Cambridge

Denoel M, Ficetola G (2014) Heterochrony in a complex world: disentangling environmental processes of facultative paedomorphosis in an amphibian. J Anim Ecol 83:606-615

Ebenman B (1987) Niche differences between age classes and intraspecific competition in age-structured populations. J Theor Biol 124:25-33

Ebenman B (1992) Evolution in organisms that change their niches during the life cycle. Am Nat 139:990-1021

Ebenman B, Johansson A, Jonsson T, Wennergren U (1996) Evolution of stable population dynamics through natural selection. Proc R Soc Lond B 263:1145-1151

Ferriere R, Gatto M (1995) Lyapunov exponents and the mathematics of invasion in oscillatory or chaotic populations. Theor Pop Biol 48:126-171

Geffen AJ, van der Veer HW, Nash RDM (2007) The cost of metamorphosis in flatfishes. J Sea Res 58(1):35-45

Grant A (1997) Selection pressure on vital rates in density-dependent populations. Proc R Soc Lond B 264:303-306

Haldane JBS (1932) The time of action of genes, and its bearing on some evolutionary problems. Am Nat 66:5-24

Hentschel BT (1999) Complex life cycles in a variable environment: predicting when the timing of metamorphosis shifts from resource dependent to developmentally fixed. Am Nat 154:549-558

Jonsson A, Ebenman B (2001) Are certain life histories particularly prone to local extinction? J Theor Biol 209(4):455-463

Lande R (1993) Risk of population extinction from demographic and environmental stochasticity and random catastrophes. Am Nat 142:911-927
Lande R, Orzack SH (1988) Extinction dynamics of age-structured populations in a fluctuating environment. Proc Natl Acad Sci 85:7418-7421

Laudet V (2011) The origins and evolution of metamorphosis in vertebrates. Curr Biol 21:726-737

Metz J, Nisbet R, Geritz S (1992) How should we define fitness for general ecological scenarios? Trends Ecol Evol 7:198-202

McMahon DP, Hayward A (2016) Why grow up? A perspective on insect strategies to avoid metamorphosis. Ecol Entomol 41:505-515

Moran NA (1994) Adaption and constraint in complex life cycles of animals. Annu Rev Ecol Syst 25:573-600

Parks AL, Parr BA, Chin J, Leaf DS, Raff RA (1988) Molecular analysis of heterochronic changes in the evolution of direct developing sea urchins. J Evol Biol 1:27-44

Pfister CA (1998) Patterns of variance in stage-structured populations: evolutionary predictions and ecological implications. Proc Natl Acad Sci USA 95:213-218

Phillips PC (1998) Genetic constraints at the metamorphic boundary: morphological development in the wood frog, Rana sylvatica. $\mathrm{J}$ Evol Biol 11:453-463

Roff DA (1992) The evolution of life histories. Theory and analysis. Chapman \& Hall, London

Rowe L, Ludwig D (1991) Size and timing of metamorphosis in complex life cycles: time constraints and variation. Ecology $72: 413-427$

Ruokolainen L, Lindén A, Kaitala V, Fowler MS (2009) Ecological and evolutionary dynamics under coloured environmental variation. Trends Ecol Evol 24:555-563

Saether B-E, Bakke O (2000) Avian life history variation and contribution of demographic traits to the population growth rate. Ecology 81:642-653

Saether B-E, Engen S (2015) The concept of fitness in fluctuating environments. Trends Ecol Evol 30:273-281

Schoener TW (1973) Population growth regulated by intraspecific competition for energy or time: some simple representations. Theor Popul Biol 1:56-84

Sherratt E, Vidal-Garcia M, Anstis M, Keogh JS (2017) Adult frogs and tadpoles have different macroevolutionary patterns across the Australian continent. Nat Ecol Evol 1:1385-1391

Strathmann RR (1978) The evolution and loss of feeding larval stages of marine invertebrates. Evolution 32:894-906

Szarski H (1957) The origin of the larvae and metamorphosis in Amphibia. Am Nat 91:283-301

ten Brink H, de Roos AM, Dieckmann U (2019) The evolutionary ecology of metamorphosis. Am Nat 193:E116-E131

ten Brink H, Onstein RE, de Roos AM (2020) Habitat deterioration promotes the evolution of direct development in metamorphosing species. Evolution 74:1826-1850

Truman J, Riddiford L (1999) The origin of insect metamorphosis. Nature 401:447-452

Tuljapurkar, (1982) Population dynamics in variable environments III. Evolutionary dynamics of r-selection. Theor Popul Biol 21:141-165

Tuljapurkar SD (1990) Delayed reproduction and fitness in variable environments. Proc Nat Acad Sci 87:1139-1143

Tuljapurkar SD, Orzack SH (1980) Population dynamics in variable environments I. Long-run growth rates and extinction. Theor Popul Biol 18:314-342

Wassersug RJ (1975) The adaptive significance of the tadpole stage with comments on the maintenance of complex life cycles in anurans. Am Zool 15:405-417 
Watkins TB (2001) A quantitative genetic test of adaptive decoupling across metamorphosis for locomotor and life-history traits in the Pacific tree frog, Hyla regilla. Evolution 55:1668-1677

Werner EE (1986) Amphibian metamorphosis: growth rate, predation risk, and optimal size at transformation. Am Nat 128:319-341

Werner EE (1988) Size, scaling, and the evolution of complex life cycles. In: B. Ebenman B., and L. Persson, (ed) Size structured populations: ecology and evolution. Springer, Berlin, pp 60-81

Wiens JJ, Kuczynkski CA, Duellman WE, Reeder TW (2007) Loss and re-evolution of complex life cycles in marsupial frogs: does ancestral trait reconstruction mislead? Evolution 61:1886-1899
Wilbur HM (1980) Complex life cycles. Annu Rev Ecol Syst 11:67-93

Wilbur HM, Collins JP (1973) Ecological aspects of amphibian metamorphosis. Science 182:1305-1314

Wray GA, Raff RA (1991) The evolution of developmental strategy in marine invertebrates. Trends Ecol Evol 6:45-50 